\title{
Spatial Impact Network Exposure Model (SINEM) with Integration of Traffic Characteristics and Air Pollution Concentration
}

\author{
Sumanta Ghosh \\ s.ghosh@qdcqatar.net / sumantaghosh.76@gmail.com \\ Qatar Design Consortium, Doha, Qatar \\ Rohit Rp \\ rohit.rp@qdcqatar.net / rohitrp.planner@gmail.com \\ Qatar Design Consortium, Doha, Qatar.
}

\begin{abstract}
The State of Qatar has undergone rapid economic growth and urbanization during the last few decades. This has resulted in an increase in the number of motor vehicles in the country. Therefore, it is vital to monitor and manage the air pollution level in Qatar. Trafficrelated air pollutants generally have a significant contribution to air pollution especially in urban areas and they also have an adverse impact on the commuters and abutting street population. Limited study has been carried out in this area and particularly in the context of Qatar. The purpose of this research is to establish a relationship between air pollution concentration and a set of parameters related to traffic and network characteristics; such as traffic volume, speed, VCR, etc. This study aims to develop an area-wide Spatial Impact Network Exposure Model (SINEM) using Geographic Information System (GIS). The SINEM shall be developed to assess the area-wide spatial impact of trafficrelated air pollution exposure based on an aggregated approach. This model helps to predict the surrounding network exposure index which helps in the decision-making process. Interestingly, this model also has the potential to scale up as well as convert into a real-time model. It will assist relevant authorities and decision-makers to identify and initialize policy measures to reduce the exposure impact, setting up building regulations, FAR implementation and sustainable master plan development.
\end{abstract}

Keywords: Traffic pollution; Air pollution; Exposure impact; Spatial assessment; Urban air quality

\section{INTRODUCTION}

Traffic related air pollution is one of the most significant problems that targets a large number of urban areas (Gwilliam et al., 2004). In many areas, motor vehicle air pollutants such as black carbon (BC), carbon monoxide (CO), nitric oxides (NOx), and particulate matter (PM) have become a dominant source of air pollution (Liu et al., 2019). The increasing congestion and vehicular traffic growth may greatly increase the pollutant emission particularly near the roadways (Zhang \& Batterman, 2013).

Air pollution is one of the major challenge for the state of Qatar especially with the upcoming events such as FIFA World Cup 2022 etc. (Charfeddine et al., 2016). Over last few decades, Qatar has been through an accelerated phase of urbanization which has come with a drastic increase in the number of motor vehicles on the nation's roads. The number of registered motor vehicles in Qatar have increased nearly 2.9 times over 
a decade (Refer to Figure 1). Therefore, the proper monitoring and management of the air pollution level in Qatar is a necessity. Major initiatives needs to be taken in order to ensure real progress in the environment sustainability pillar as part of Qatar Vision 2030.

\section{No. of Registered Vehicles}

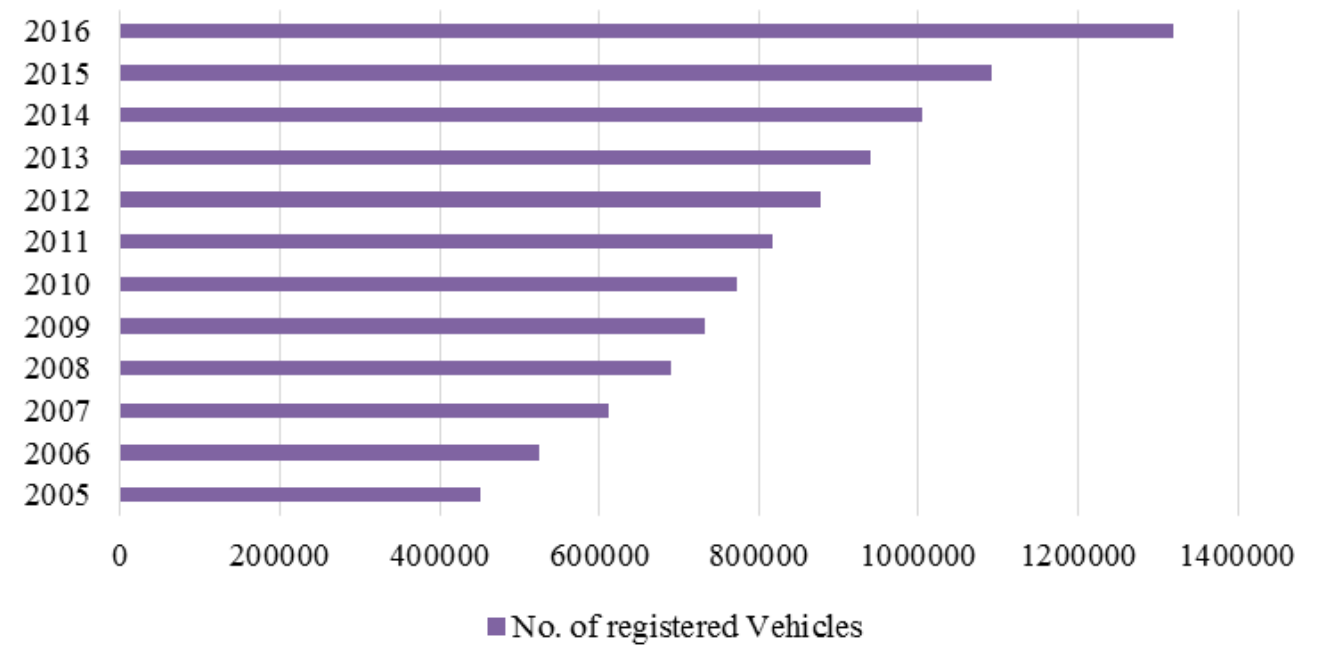

Figure 1: Number of registered motor vehicles (Source: Qatar Planning and Statistics Authority)

Traffic-related air pollutants generally have a significant contribution to air pollution especially in urban areas and have an adverse impact on the commuters and abutting street population. There are several literatures available to investigate the functional relationship between air quality and traffic related air pollution (Kumar et al., 2015). Most research papers that are available either lack in their ability to relate air pollution exposure to traffic characteristics, or they are too complex and not cost effective. Additionally, majority of the research is for exposure appreciation rather than prediction. Limited study has been carried out in this area particularly in the context of Qatar. This paper intends to develop a model framework to establish a relationship between air pollutant concentration and a set of parameters related to traffic and network characteristics; such as traffic volume, speed, vehicle capacity ration (VCR), etc. In the current scenario, implementing monitoring stations at multiple locations within the city are costly and not economically viable. There is a potential opportunity for the integration of the existing available data sources and currently available traffic prediction models such as the Qatar Strategic Transport Model (QSTM) from Ministry of Transportation \& Communication (MOTC). The framework presented in this paper aims to develop a prediction model which is an area-wide Spatial Impact Network Exposure model (SINEM) using Geographic Information System (GIS). This model will have the capability to predict the surrounding network exposure index, which assists in the decision-making process and sustainable planning approach. 
The paper is organized as follows: Section 1 introduced with the context of the research; Section 2 provides the brief review of the literatures on traffic related air pollution modelling; In Section 3 core elements of the SINEM framework has been explained through schematic flowchart; Section 4 details out the different stages of the SINEM framework with existing data evaluation in GIS platform, model building, prediction and spatial assessment in GIS; Section 5 presents the model benefits and possible interpretations; Section 6 gives the various conclusion of the paper.

\section{LITERATURE REVIEW}

Sexton and Ryan (1988) has carried out an extensive research on the air pollution exposure models and methods. It is stated that specific emissions sources to the individual exposure are necessary and new models to be developed. However, very few literatures are available for a predictive model for street exposure in particular to traffic related air pollutions. Most of the research works carried out to model the traffic related air pollution and exposure models are limited to pollution concentration, street typology (micro level), specific age group and building height (for example: (Askariyeh et al., 2019); (Valente \& Amorim, 2014). However, the congestion period, v/c ratio, speed relation etc., are not taken into account in any of these models. Application of the GIS in air pollution modelling has been carried out by previous researchers (Matejicek, 2005). Considering the specific requirement such as the amount of data, software expertise, implementation cost and marginal benefits, GIS based hybrid model serves the purpose of the exposure modelling at an aggregate level (Jerrett et al., 2005). In context of Qatar, integration of the existing QSTM traffic model with pollution concentration can be better analyzed, and prediction models can be developed which are cost effective and not complex in nature. Also, it is worth mentioning that dynamic prediction models with long term series of data can be better trained with an artificial neural network for improved prediction accuracy (for example (Karimian et al., 2019), (Cabaneros et al., 2017).

\section{SPATIAL IMPACT NETWORK EXPOSURE MODEL (SINEM) FRAMEWORK}

As shown in the Figure 2, the core elements of the SINEM framework include multiple stages. Initial stages define the spatial and input definition. Spatial definition represents the spatial extent of the model. Input definition defines the dependent (pollution exposure) and independent variables (traffic, environment and study area specific parameters) to be considered for the model. As part of the existing/baseline study, these input variables are to be evaluated and assessed through statistical test to establish the correlation between the dependent and independent variables. 


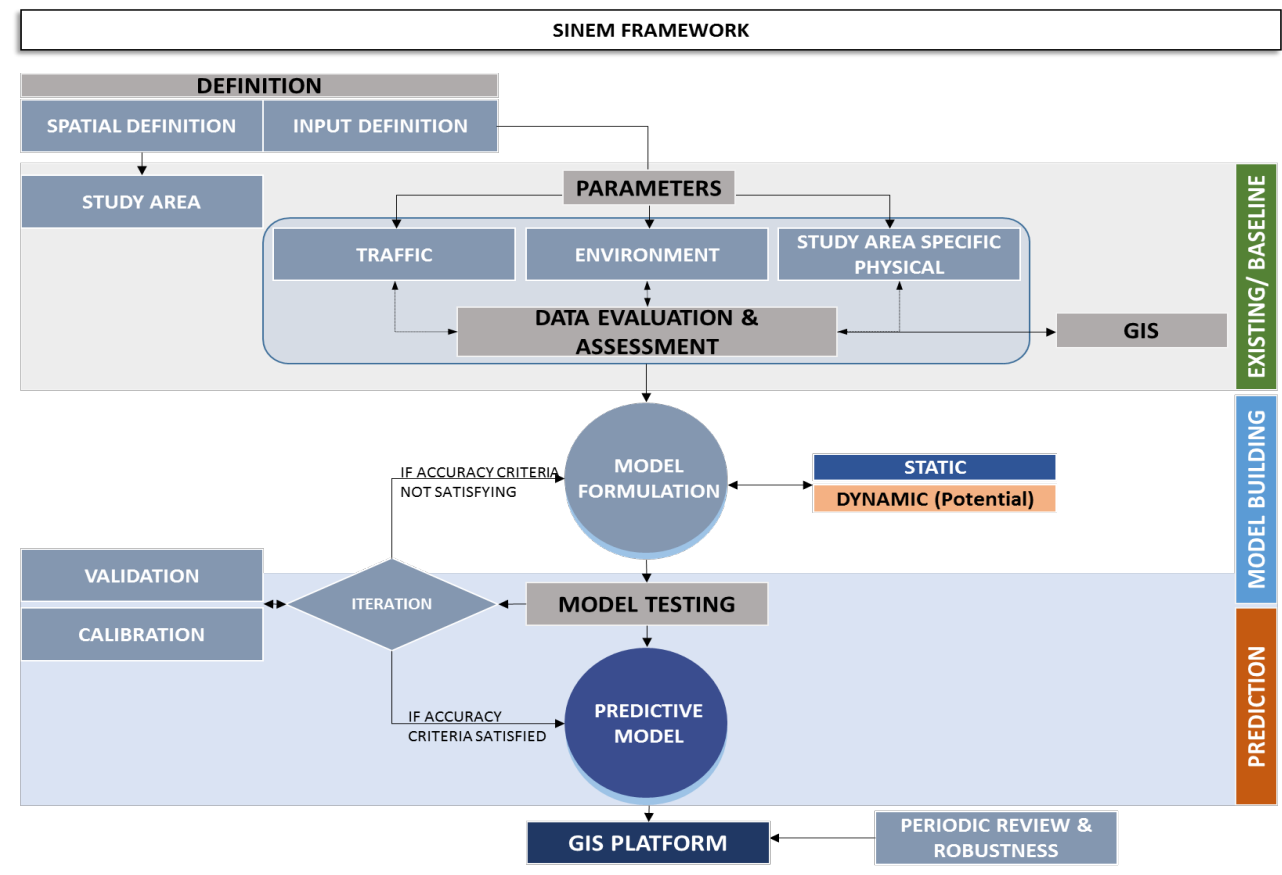

Figure 2: Core Elements of SINEM Framework

GIS has been considered as part of the model building since the digital maps and citywide data base are available for whole of Qatar. ArcGIS software to be utilized for this purpose. As part of the model building process, spatial extent of the study area to be converted into a grid form with $100 \mathrm{~m}$ by $100 \mathrm{~m}$ (for city level). According to Shahraiyni, the grid size for the spatial prediction in urban areas should not be less than $100 \mathrm{~m}$ (Shahraiyni \& Sodoudi, 2016). Independent variables are fed into the ArcGIS platform for the entire respective grid. However, the pollution concentration data will be an input to those grids which fall under the survey locations. These grids are to be termed as active grids which inherit the properties of the existing data points. In model building, exposure estimation will primarily be carried out through a static approach using the existing pollution concentration data, as well as both the in-vehicle population and the abutting street population (please refer to Section 4.3). Utilizing the estimated exposure as a dependent variable, multiple regression models shall be developed through an iterative process to attain model accuracy. Training and testing of the model shall be carried out to improve the model prediction accuracy. If the accuracy criteria are satisfied, then the predictive model is to be integrated in the GIS platform to predict the exposure values for those inactive grid cells. It is worth mentioning that in case of a huge data set availability of the pollution concentration, this model can be scaled up to be a dynamic model through application of artificial neural network for improved accuracy and predictions (for example: Karimian et al., 2019). 


\section{STAGES OF SINEM FRAMEWORK}

This section briefly describe the stages involved in the SINEM framework. The prime aim of the model is to establish a relationship between the traffic related air pollution and pollution concentration through an indirect approach and thereby build a predictive model for the population exposure. It can be assessed and visualized in GIS platform (namely ArcGIS), and assist in spatial decision-making.

\subsection{Stage 1: Definition \& Data Collation}

The first stage is to define the spatial extent in line with the objective of the study, which delineates the study area at the scale of microscopic level (zonal level), mesoscopic (city level) or macroscopic (regional level), depending on the objective of the study. As presented in the Figure 3, input variables can be categorized in three forms namely, traffic characteristics (such as link length, traffic volume, number of lanes, v/c ratio etc.), environment (humidity, wind direction etc.) \& area specific physical features.

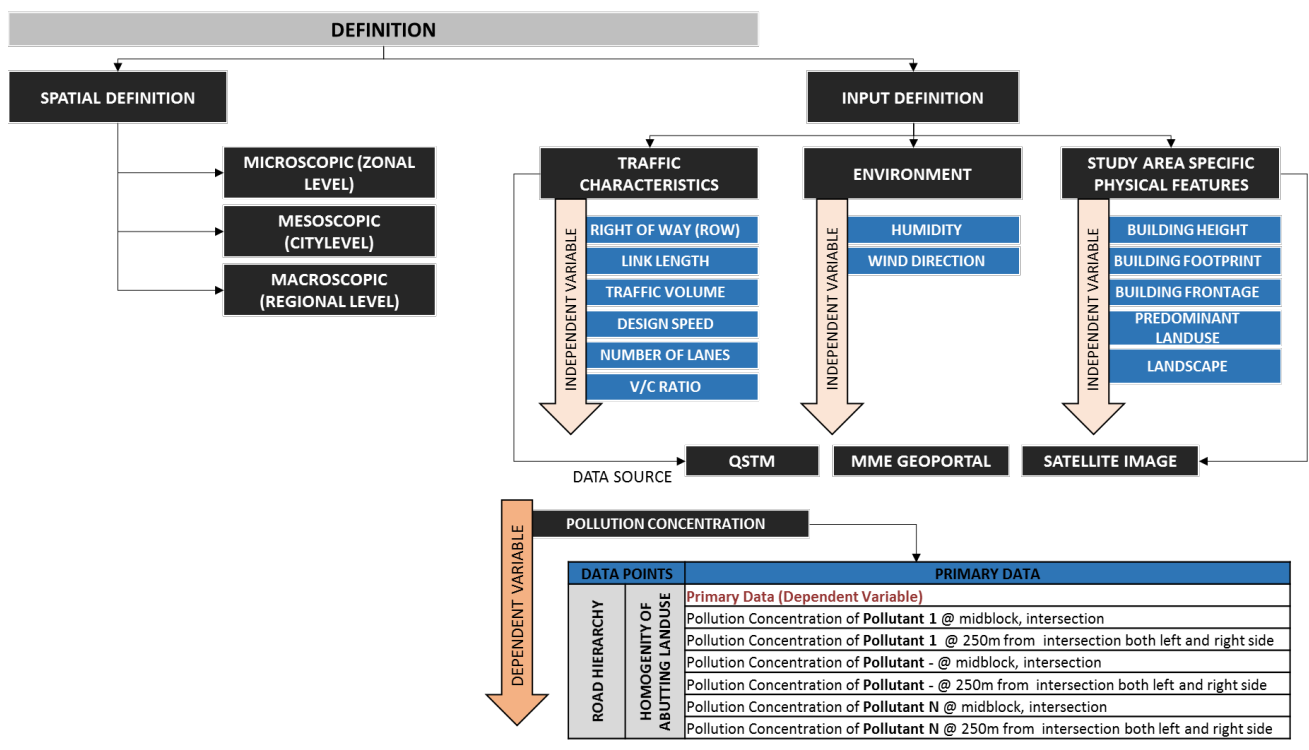

Figure 3: Stage1: Definition and Data Collation of SINEM Framework

Traffic characteristics parameters can be captured through QSTM model and this data can be easily integrated into the ArcGIS. The area specific parameters such as landscape, building footprint, building frontage etc. can be easily extracted through satellite imagery analysis in GIS platform (for example: (Dini \& Jacobsen, 2013). Land use information are available through secondary data sources such as the Ministry of Municipality and Environment (MME). However, primary data is also required for the pollution concentration levels. The data sampling is to be carried out on the basis of the road hierarchy and homogeneity of land uses to capture the discrete dataset for modelling exercise.

\subsection{Stage 2: Existing Data Assessment \& Evaluation}

Stage 2 of the framework deals with the data assessment and evaluation as presented 
in the Figure 4. Data samples of the pollution concentration of pollutants (P1, P2, Pn) collected as part of the primary survey are to be imported in the ArcGIS platform for the spatial air quality assessment and visualization. Data samples are geo-referenced in the ArcGIS overlaid with network data base link attributes which is extracted from QSTM model. Further, kriging analysis (Tyagi \& Singh, 2013) to be carried out to interpolate each pollutant's concentration data samples for the initial review. The existing data range can be classified into high, medium and low categories based on the dataset range. As explained in Section 3, the spatial grid conversion to be deployed and existing survey data points will be inherited in the particular grid cell where the data sample point located. Each grid cell will be having unique numbering and its input data attributes. The active cells with existing data points attributes are fed with all the input parameters and pollution concentration, which is used in the further steps for static model building process to estimate the street exposure.

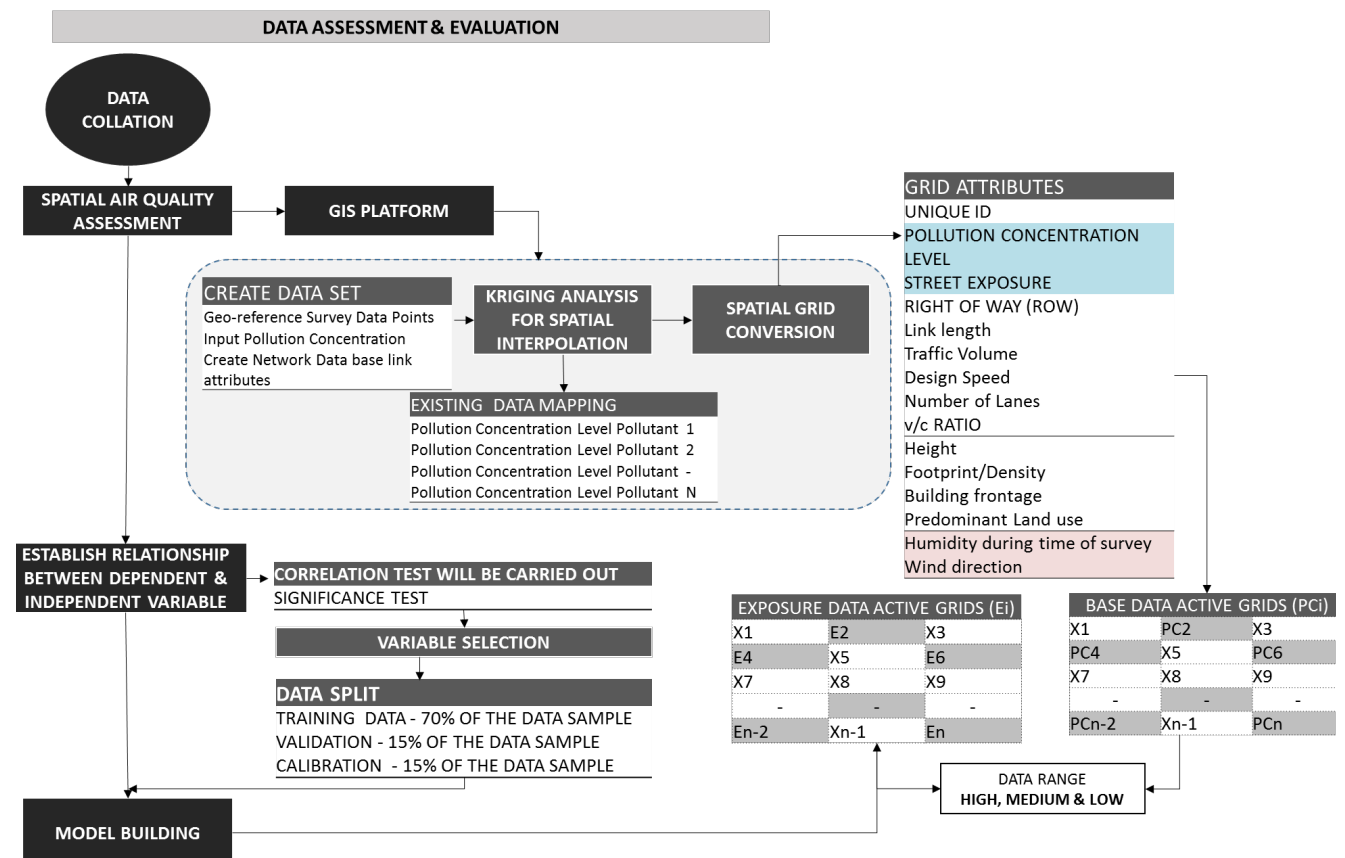

Figure 4: Stage 2: Existing Data Assessment \& Evaluation of SINEM Framework

\subsection{Stage 3: Model Building \& Prediction}

Earlier researchers such as Ott (1982), Duan (1982) established simple mathematical formulas to estimate the exposure. Simple Exposure Index does not take into account any traffic-pollution relationships. In general, a simple static exposure model is defined as the ambient air pollution at a geographic location times the total number of people at the location as presented in the equation (1) below. However, some researchers for example: (Kumar et al., 2015)) considered vehicle kilometer travelled to add traffic relation to the exposure model.

$$
\text { E index }=\mathrm{C} \text { location } * \mathrm{P} \text { location }
$$


where,

$\mathrm{C}$ location is the concentration level in street, which may be mean, percentile or max values.

$\mathrm{P}$ location is the population estimate in that particular location, which is the product of Average Daily Traffic (ADT) and vehicle occupancy.

However, in this framework, the street exposures are estimated as shown in the equation (2) below. Exposure index presented in the equation (2) is a basic mathematical expression, which is product of pollution concentration, street population and average time spent in that particular stretch.

$$
\begin{aligned}
& \text { E street }=\text { C location } * \text { Pstreet } * \frac{L}{\mathrm{~V}} * \frac{1}{\mathrm{~L}} \\
& \text { Pstreet }=(\mathrm{Nv} * \mathrm{Ov})+\text { A Pop }
\end{aligned}
$$

where,

E street is the street exposure estimate e.g. $\mu \mathrm{g} / \mathrm{m} 3 *$ person*hours $/ \mathrm{km}$.

$\mathrm{C}$ location is the concentration level in street during the peak hour.

$\mathrm{Nv}$ is the number of vehicular traffic during peak hour of type $\mathrm{v}$.

$\mathrm{Ov}$ is the vehicle occupancy for the vehicle type $\mathrm{v}$.

A Pop is the abutting street population extracted from QSTM TAZ based on population density.

$\mathrm{L}$ is the length of the street.

$\mathrm{V}$ is the travel speed of the vehicle type $\mathrm{v}$.

Pstreet is the outdoor street population affected by exposure risk. If we have the data available on pedestrian and bicycle users, can be included in the model.

Stage 3 of the framework which deals with the model building and population exposure is presented in Figure 5. As part of the model building, the exposure estimation for the street within the active grid has been calculated as per using equation (2). The active cells with attribute data to be used as an input for the exposure prediction model building. Shahraiyni \& Sodoudi (2016) have carried out an extensive comparative assessment of the different approaches for pollution prediction in urban areas. According to him, the most widely adopted technique for spatial models is the Multiple Linear Regression (MLR) model. However, to capture the temporal variations, Artificial Neural Networks (ANN) have the highest potential for better performance (Karimian et al., 2019). If a huge dataset is available to capture temporal variations, ANN can be deployed in the model for a dynamic prediction model.

In this case, MLR has been considered in the SINEM framework for static modelling for a smaller dataset. Input data is considered based on the correlation test for each pollutant exposure to be considered in the model building. Training and testing of the model shall be carried out with data split proportion of $70 \%$ and $30 \%$ of the data samples respectively (Dobbin \& Simon, 2011). The observed and estimated data has to be tested using statistical approaches such as R squared, the overall F-test and the Root Mean Square Error (RMSE) value for the scatterplot observed and estimate values to evaluate the model fit. If the model fails the test then the model needs to be remodeled with the 
best fit function and changes in the parameter coefficient, if required. The predictive model developed needs to be integrated in the ArcGIS to predict the exposure index for the inactive cells. Thus, the model will be capable of fitting the different scenarios, or with the change in the parameters such as ROW, number of lanes, vehicle volume etc. effect on the street population exposure can be estimated and assessed for decisionmaking.

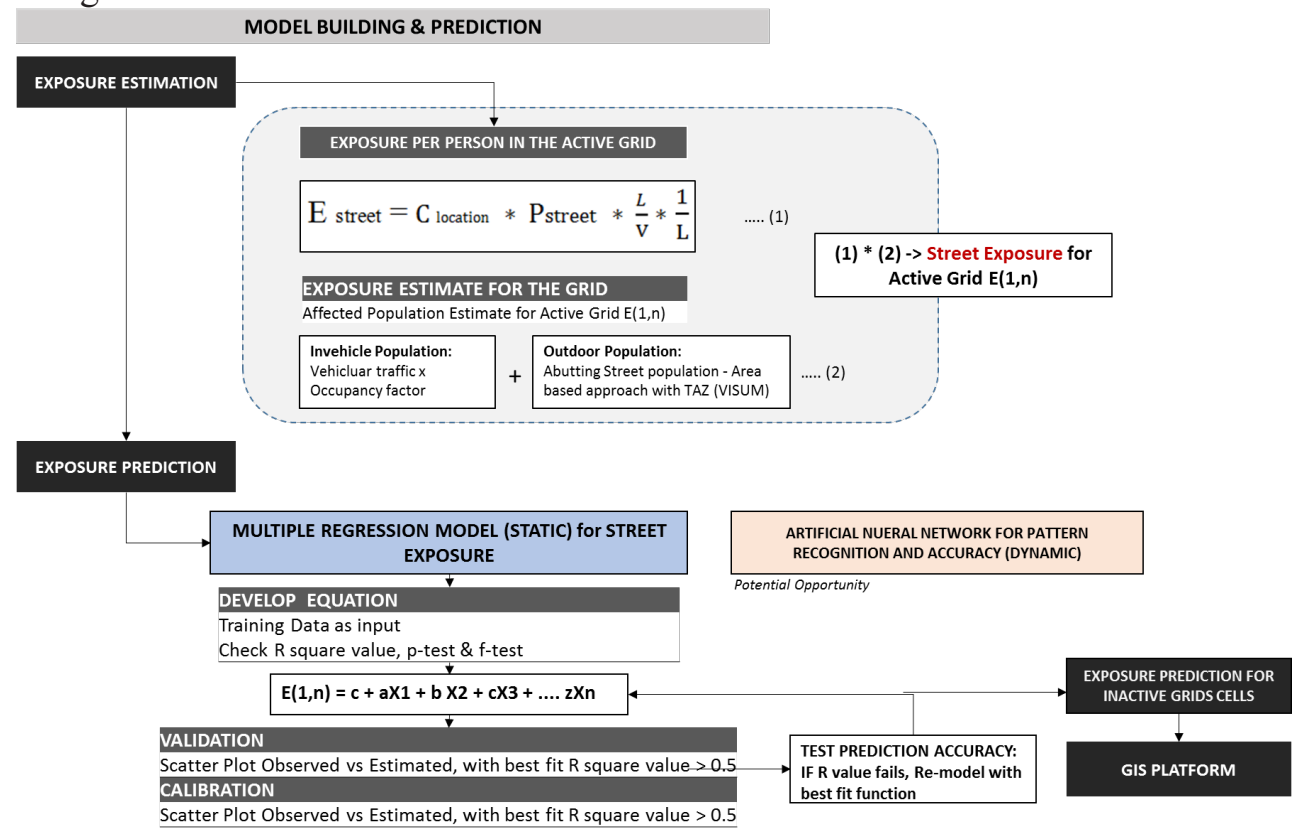

Figure 5: Stage 3: Model Building and Prediction of SINEM Framework

\section{ANTICIPATED RESULTS}

The key benefits of the SINEM framework are presented below:

a) SINEM helps to understand the exposure levels variation in between and within the different street/network typology.

b) It helps to identify the relationship between each pollutant concentration (traffic related) and traffic/network characteristics.

c) It is a static model in nature but interestingly it can be scaled up to be a dynamic model with increased prediction accuracy using ANN.

d) Allows for the mapping of pollution concentration and exposure level.

e) It has the ability to test different scenarios, which assist in the master planning exercise, policy formulation, building byelaws formulation, FAR implementation and decision making to ensure a sustainable development.

\section{CONCLUSION}

In the context of the current urbanization rates and increase in the number of vehicles, traffic-related pollution contributes to serious health issues for citizens. It is therefore necessary to monitor and evaluate the traffic-pollution relationships and its spatial impact on the population exposure. In the state of Qatar, majority of the population resides in urban areas. Implementing monitoring stations at multiple locations within 
the city to monitor the air pollution levels are not a feasible solution as they are costly and not economically viable. Therefore, the development of a predictive model is crucial for the welfare of society.

This paper presents the integrated modelling framework involving GIS for both exposure assessment and prediction spatially for traffic related air pollutants. The study aims to develop an area-wide Spatial Impact Network Exposure Model (SINEM) integrating traffic-related air pollution using Geographic Information System (GIS). It is a static model integrated into the ArcGIS platform. It makes use of the existing data resources such as satellite imagery, QSTM model, MME GIS portal etc. to capture the input parameters, and thereby it is economically viable and not complex. With the increase of data availability in the future, it can be scaled up to be a dynamic model with the application of ANN for improved accuracy.

\section{REFERENCES}

Askariyeh, M. H., Vallamsundar, S., Zietsman, J. \& Ramani, T. (2019). Assessment of trafficrelated air pollution: Case study of pregnant women in South Texas. International Journal of Environmental Research and Public Health, 16 (13), 2433.

Cabaneros, S. M., Calautit , J. K. \& Hughes , B. R. (2017). Hybrid artificial neural network models for effective Prediction. Energy Procedia. 142, 3524-3530.

Dini, R. \& Jacobsen, K. (2013). Delineation of building footprints from high resolution satellite stereo imagery using image matching and a GIS database. International Archives of the Photogrammetry, Remote Sensing and Spatial Information Sciences, XL-1/W1, 81-85.

Dobbin, K. K. \& Simon, R. M. (2011). Optimally splitting cases for training and testing high dimensional classifiers. BMC Medical Genomics, 4, 31.

Duan, N. (1982). Models for human exposure to air pollution. Environment International, 8, 305-309.

Gwilliam, K., Kojima, M. \& Johnson, T. (2004). Reducing Air Pollution from Urban Transport. Washington, D.C.: The World Bank.

Jerrett, M., Arain, A., Kanaroglou, P. \& Beckerman, B. (2005). A review and evaluation of intraurban air pollution exposure models. Journal of exposure analysis and environmental epidemiology, 15, 185-204.

Karimian, H., Li, Q., Wu, C., Qi, Y. \& Mo, Y. (2019). Evaluation of different machine learning approaches to forecasting PM2.5 mass. Aerosol and Air Quality Research, 19, 1400-1410.

Kumar, A., Mishra, R. K. \& Singh, S. (2015). GIS application in urban traffic air pollution exposure. Suan Sunandha Science and Technology Journal, 2, 25-37.

Lanouar, C., Al-Malk, A. Y. \& Al Karbi, K. (2016). Air Pollution in Qatar : Causes and Challenges. College of Business and Economics, Qatar University, 1, 1-7.

Liu, S. V., Chen, F.-1. \& Xue, J. (2019). A meta-analysis of selected near-road air pollutants based on concentration decay rates. Heliyon, 5 .

Matejicek, L. (2005). Spatial modelling of air pollution in urban areas with GIS: A case study on integrated database development. Advances in Geosciences, 63-68.

Ott, W. R. (1982). Concepts of human exposure to air pollution. Environment International, 7 , 179-196. 
Sexton, K. \& Ryan, P. (1988). Assessment of Human Exposure to Air Pollution: Methods, Measurements, and Models. In W. AY, B. RR, \& K. D, Air Pollution, the Automobile, and Public Health. Washington (DC): National Academies Press (U.S).

Shahraiyni, H. T. \& Sodoudi, S. (2016). Statistical Modeling Approaches for PM10 Prediction. Atmosphere, 7, 10-13.

Tyagi, A. \& Singh, P. (2013). Applying Kriging Approach on Pollution Data Using GIS Software. International Journal of Environmental Engineering and Management, 4, 185-190.

Valente, J., Amorim, J. H., Teixeira, R., Pimentel, C. \& Borrego, C. (2014). Children's exposure to traffic-related pollution: assessment of $\mathrm{CO}$ exposure in a typical school day. International Journal of Environment and Pollution (IJEP), 55, 104-112.

Zhang, K. \& Batterman, S. (2013). Air pollution and health risks due to vehicle traffic. Science of The Total Environment. 450, 307-316. 